\title{
The relationship between lymphocyte subsets and clinico-pathological determinants of survival in patients with primary operable invasive ductal breast cancer
}

\author{
Z M A Mohammed ${ }^{*}, 1,2,3$, J J Going ${ }^{2}$, J Edwards ${ }^{4}$, B Elsberger ${ }^{5}$ and D C McMillan ${ }^{1}$ \\ ${ }^{1}$ Academic Unit of Surgery, College of Medical, Veterinary and Life of Sciences- University of Glasgow, Royal Infirmary, Glasgow \\ G31 2ER, UK; ${ }^{2}$ University Departments of Pathology, College of Medical, Veterinary and Life of Sciences- University of Glasgow, \\ Southern General Hospital, Glasgow, UK; ${ }^{3}$ University Departments of Pathology, Faculty of Veterinary Medicine, Omar Almukhtar \\ University, Al bayda, Libya; ${ }^{4}$ Unit of Experimental Therapeutics, Institute of Cancer, College of Medical, Veterinary and Life \\ Sciences, Western Infirmary, Glasgow, UK and ${ }^{5}$ Surgical Oncology, University of Dundee, Ninewells Hospital, Dundee, UK
}

Background: The importance of lymphocyte subtypes in determining outcome in primary operable ductal invasive breast cancer remains unclear. The aim of present study was to examine the relationship between tumour lymphocyte subsets infiltrate and standard clinico-pathological factors and survival in patients with primary operable invasive ductal breast cancer.

Methods: The analysis of the inflammatory cell infiltrate, including lymphocyte subtypes, was undertaken using immunohistochemical techniques and visual quantitative and semi-quantitative techniques in 338 patients with ductal breast cancer.

Results: The majority (91\%) of patients had high grade inflammatory cell infiltrate. The median follow-up of the survivors was 164 months. During this period, 65 died of their cancer. On univariate analysis, tumour inflammatory cell infiltrate, macrophages infiltrate $(P<0.05)$, lymphocytic infiltrate $(P<0.001)$ and CD8 + T-lymphocytic infiltrate $(P<0.01)$ were associated with improved cancer-specific survival, whereas neutrophil $(P<0.05)$ and CD138 + B-lymphocytic infiltrate $(P<0.001)$ were associated with poorer cancer-specific survival. On multivariate analysis, tumour lymphocytic infiltrate $(P<0.001)$, macrophage infiltrate $(P<0.05)$, CD8 + T-lymphocytic infiltrate $(P<0.01)$ and CD138 + B-lymphocytic infiltrate $(P<0.001)$ were independently associated with cancer survival. When the significant inflammatory cell types were included with tumour-based factors in multivariate analysis only tumour size (Hazard ratios (HR): 2.55, 95\% confidence interval $(\mathrm{Cl}): 1.53-4.27, \mathrm{P}<0.001)$, Ki-67 index (HR: 2.08, 95\% Cl: 1.08-4.00, $P<0.05)$, lymphovascular invasion (HR: 4.40, 95\% Cl: 2.07-9.35, $P<0.001)$, macrophage infiltrate (HR: 0.49, 95\% $\mathrm{Cl}$ : 0.33-0.73, $P<0.001)$, lymphocytic infiltrate (HR: $0.11,95 \% \mathrm{Cl}: 0.05-0.23, P<0.001)$, CD8+ T-lymphocytic infiltrate (HR: 0.57, 95\% Cl: 0.38-0.87, $P<0.001)$ and CD138+ B-lymphocytic infiltrate (HR: 2.86, 95\% Cl: 1.79-4.56, $P<0.001)$ were independently associated with cancer survival.

Conclusion: The majority of patients with invasive ductal breast cancer had high-grade inflammatory cell infiltrate. In these patients, inflammatory cells including macrophage and lymphocytic infiltrate, and subsets CD8+ T-lymphocytic infiltrate and CD138 + B-lymphocytic infiltrate had superior prognostic value, compared with hormone status and lymph node involvement in patients with primary operable invasive ductal breast cancer.

*Correspondence: ZMA Mohammed; E-mail: z.mohammed.1@research.gla.ac.uk and mrxzma@hotmail.com

Received 7 May 2013; revised 22 July 2013; accepted 26 July 2013; published online 27 August 2013

(C) 2013 Cancer Research UK. All rights reserved 0007-0920/13 
Breast cancer is the commonest cancer and the leading cause of cancer death in women. It accounts for $22 \%$ of all female cancers (Parkin et al, 2001). More than 42000 women in the United Kingdom are diagnosed with breast cancer each year and $\sim 80 \%$ survive at least 5 years (Cancerstats, 2011).

The prognosis of breast cancer is influenced by wellrecognised host- and tumour-related factors including patient age, histological type and grade, tumour size, lymph node status, oestrogen receptor (ER) and progesterone receptor (PR) status, and human epidermal growth factor receptor 2 (HER-2) status (Lal et al, 2005).

There is now good evidence that the development of cancer and its progression are dependent of a complex interaction of the tumour and the host inflammatory response (Vakkila and Lotze, 2004; DeNardo and Coussens, 2007; Colotta et al, 2009; Hanahan and Weinberg, 2011). In particular, the local inflammatory response represents the interaction between the tumour and the host. A number of inflammatory cell types have been implicated in this response (Mohammed et al, 2012c). For example, our previous work using simple routine pathology haematoxylin and eosin (H\&E) slides showed that lymphocytes were the most prominent cells infiltrating and had independent prognostic value in patients with primary operable invasive ductal breast cancer. In contrast, plasma cells (subtype of B lymphocytes) were associated with poorer survival (Mohammed et al, 2012b).

However, despite the large number of studies and considerable effort over a 50-year period, the relationship between different aspects of lymphocyte subsets infiltrate and outcome in primary operable breast cancer remains unclear. Although a number of studies over these years have attempted to examine these relationships, there have been conflicting results. For example, out of a total of 18 studies, 5 studies reported an association between T-lymphocyte subsets infiltrate and improved survival, 4 reported no association and 9 reported an association between T-lymphocytes subsets and poorer survival (Mohammed et al, 2012c). With reference to B lymphocytes, out of a total of 7 studies, 4 studies reported an association between B-lymphocyte subsets infiltrate and improved survival, 2 reported no association and 1 reported an association between B-lymphocyte subsets and poorer survival (Mohammed et al, 2012c).

An important potential confounder in previous work is that the immune context has rarely been taken into account. For example, where there is a dense tumour inflammatory cell infiltrate, the presence of a variety of inflammatory cell types is likely to reflect the activity of a coordinated immune response. In contrast, where there is a sparse inflammatory cell infiltrate, the presence of inflammatory cell types is likely to be associated with a different immune response. To date, few studies have considered this in their analysis of the relationship between individual inflammatory cell types and outcome.

Therefore, in this context, it is of considerable interest to examine lymphocytic infiltration using more sophisticated techniques to better understand the nature of such relationship between the tumour infiltrate and breast cancer outcome. Most tumour infiltrating lymphocytes are recognised to be of the CD3+ T-lymphocyte phenotype. This phenotype includes tumour CD4+ T lymphocytes and CD $8+$ cytotoxic T lymphocytes. In the normal state, $\mathrm{CD} 4+$ is expressed on $\sim 60 \%$ of mature $\mathrm{T}$ lymphocytes, whereas $\mathrm{CD} 8+$ is expressed on $30 \%$ of mature $\mathrm{T}$ lymphocytes giving an approximate $2: 1$ ratio.

Also, B lymphocytes have an important role in the humoral immune response and are responsible for both antigen presentation as well as antibody production. A common marker used to assess B lymphocytes is CD20 + that is expressed on B-lymphocyte precursors and mature $\mathrm{B}$ lymphocytes but lost during terminal plasma cell differentiation (Tedder and Engel, 1994), whereas monoclonal antibodies of the CD138 + have been developed and appear to be plasma cell-specific among hematopoietic elements (Costes et al, 1999).

The aim of the present study was to examine the relationship, in the context of low- and high-grade generalised inflammatory cell infiltrate, between tumour lymphocyte subset infiltrate and standard clinico-pathological factors including hormone status (ER, PR and HER-2), Ki-67, lymphovascular invasion and survival in patients with primary operable invasive ductal breast cancer. In particular, to test the hypothesis that different lymphocyte subsets would have a different relationship with long-term outcome.

\section{PATIENTS AND METHODS}

Patients presenting with invasive ductal breast cancer at Royal Infirmary, Western Infirmary and Stobhill Hospital, Glasgow, between 1995 and 1998 were studied $(n=338)$. Clinico-pathological data included age, histological tumour type, grade, tumour size, lymph node status, lymphovascular invasion, type of surgery and use of adjuvant treatment (chemotherapy, hormonal therapy and radiotherapy) were retrieved from the routine reports.

In the present study, a consecutive series (including symptomatic non-screened patients) were examined. As a result many of the cancers were relatively advanced, and the proportions of grade III and node positive cancers were relatively high. Also, the patients included in this study did not receive neoadjuvant therapy or adjuvant anti-HER-2 therapy.

Oestrogen receptors (ER), progesterone receptors (PR), HER-2 status and Ki-67 immunohistochemical analyses were performed as previously described, and the results of visual scoring were used in this study (Mohammed et al, 2012a,d,e). The inclusion of ductal breast cancers only was to limit the potential confounding effects of other tumour types on the analysis in the present study.

Institutional Review Board approval for use of human tissue in this study was given by the Research Ethics Committee of the North Glasgow University Hospitals NHS Trust.

\section{Methods}

Tissue micro array construction. Tissue microarrays (TMA) were used in this study. The TMA samples were taken from the tumourrich area of each specimen, and TMAs were constructed in triplicate, using $0.6 \mathrm{~mm}^{2}$ cores, to account for intra-tumour disease heterogeneity as previously described (Mohammed et al, 2012e).

Slide scanning and scoring. Stained slides were scanned at objective magnification $\times 40$ using a Hamamatsu NanoZoomer (Hertfordshire, UK). Assessment of tumour inflammatory cell infiltrate was performed on a computer monitor using Slidepath Tissue IA system version 3.0 (Dublin, Ireland).

Assessment of tumour inflammatory infiltrate. Full-section haematoxylin and eosin slides for the 338 patients were used to score peri-tumoural inflammatory cell infiltrate according to Klintrup criteria. Klintrup scoring of slides was carried out as previously described. Briefly, tumours were scored on a 4-point scores based on appearances at the edge of tumour invasion. A score of 0 signified that there were no inflammatory cells at tumour's invasive margin; score 1 , indicated a mild and patchy inflammatory cells; score 2, denoted a prominent band-like inflammatory reaction at the invasive margin and score 3 , revealed a florid cup-like inflammatory infiltrate at the invasive edge with frequent destruction of cancer cell islands (Klintrup et al, 2005). Full-section haematoxylin and eosin slides were also used to score the proportions of neutrophils and lymphocytes as previously reported. Briefly, neutrophils and lymphocytes were estimated in a 
minimum of 4 to a maximum of 12 fields $(\times 40,0.6 \mathrm{~mm}$ in diameter) at the invasive margin on full-section haematoxylin and eosin slides (Mohammed et al, 2012b). The Klintrup criteria were described as absent or present. In light of specific staining of macrophages and lymphocytes subsets, this is now termed lowand high-grade Klintrup criteria, respectively. TMA and immunohistochemistry were utilised to assess macrophages as previously reported (Mohammed et al, 2012b).

Immunohistochemistry for lymphocytes subsets. Immunoreactivity to anti-CD4 +, CD8 +, CD20 + and CD138 + antibodies was determined in 40 cases by using full-faced tissue sections. Tissue microarrays (TMAs) were also utilised to assess $\mathrm{T}$ and B lymphocyte subsets for the 338 patients. Freshly cut $2.5-\mu$ m-thick breast cancer full-faced tissue sections and TMAs were stained for $\mathrm{CD} 4+, \mathrm{CD} 8+, \mathrm{CD} 20+$ and $\mathrm{CD} 138+$. Sections and cores were dewaxed and rehydrated. Antigen retrieval for CD4 +, CD8 + and $\mathrm{CD} 138+$ were performed by keeping the section in Tris-EDTA buffer $(\mathrm{pH} 8)$ in pressure cooker for $7 \mathrm{~min}$, whereas antigen retrieval for $\mathrm{CD} 20+$ was performed by keeping the section in $10 \mathrm{mmoll}^{-1}$ sodium citrate buffer ( $\mathrm{pH}$ 6.0) in pressure cooker for $20 \mathrm{~min}$ (Mahmoud et al, 2011a). Endogeneous peroxidise was locked by incubation in $3 \%$ hydrogen peroxide for $20 \mathrm{~min}$. The cores were then incubated with the normal horse serum at dilution $1: 10$ for $30 \mathrm{~min}$ at $25^{\circ} \mathrm{C}$ to block non-specific binding sites. The primary antibodies for $\mathrm{CD} 4+$ (monoclonal mouse anti-human, CD4 +, Clone 4B12, Code VP- C319, Vector, Peterborough, UK) added with dilution $1: 10$ for $30 \mathrm{~min}$ at $25^{\circ} \mathrm{C}$, for $\mathrm{CD} 8+$ (monoclonal mouse anti-human, CD8 +, Clone CD8/ 144B, Code M 7103, DAKO, Glostrup, Denmark) added with dilution 1:400 at $4{ }^{\circ} \mathrm{C}$ for overnight, for CD20 + (monoclonal mouse anti-human, CD29cy, Clone L26, Code M0755, Dako, Glostrup, Denmark) added with dilution $1: 300$ for $30 \mathrm{~min}$ at $25^{\circ} \mathrm{C}$ and that for $\mathrm{CD} 138+$ (monoclonal mouse anti-human, CD138 +, Clone MI15, Code M 7228, DAKO, Glostrup, Denmark) added with dilution $1: 100$ at room temperature for $30 \mathrm{~min}$. Sites of binding were detected using the Envision technique (DAKO code K5007, Glostrup, Denmark) with DAB (3-3' diaminobenzidine, Vector code SK 4001, USA), a chromogenic substrate, according to the manufacturer's instruction. Cores were counterstained with haematoxylin, dehydrated and mounted with DPX. Two full sections of the tonsil tissue were used as positive and negative controls for each set of staining, since tonsil had previously shown to have a high $\mathrm{CD} 4+, \mathrm{CD} 8+, \mathrm{CD} 20+$ and $\mathrm{CD} 138+$ content, by immunohistochemical staining.

Assessment of CD4+, CD8 +, CD20 + and CD138+ and scoring in full-faced tissue sections and TMA was based on a modification of the method described by (Mahmoud et al, 2011b; Mahmoud et al, 2011a) in which the number of $\mathrm{T}$ and B lymphocytes was counted. In the full-faced tissue sections, CD4+, $\mathrm{CD} 8+, \mathrm{CD} 20+$ and $\mathrm{CD} 138+$ were counted in three $\times 20$ fields, and the mean for the three fields was calculated. In TMA, CD4+, $\mathrm{CD} 8+, \mathrm{CD} 20+$ and $\mathrm{CD} 138+$ were counted in the three cores and, as each tumour had triplicate cores, the mean count for each carcinoma was taken as a final score. The means for the three fields in full-faced sections for CD4 +, CD8 +, CD20 + and CD138+ were compared with the mean of three cores for $\mathrm{CD} 4+, \mathrm{CD} 8+$, $\mathrm{CD} 20+$ and $\mathrm{CD} 138+$ in TMA for the same patients. The Interclass Correlation Coefficients (ICCCs) were 0.88, 0.99, 0.80 and 0.80 for $\mathrm{CD} 4+, \mathrm{CD} 8+, \mathrm{CD} 20+$ and $\mathrm{CD} 138+$, respectively, which indicates excellent agreement.

One hundred and eighty cores were independently scored for $\mathrm{CD} 4+, \mathrm{CD} 8+$ and $\mathrm{CD} 20+$ by two observers, $\mathrm{ZM}$ and BE, and for $\mathrm{CD} 138+$ by ZM and JG blinded to patient outcome and the other observer's score. The ICCCs were 0.90 for CD4,+ 0.90 for $\mathrm{CD} 8+, 0.86$ for $\mathrm{CD} 20+$ and 0.90 for $\mathrm{CD} 138+$, which indicates excellent agreement. The author (ZM) then scored all slides, and these data were used in subsequent analysis.

Patients underwent regular follow-up (3 months, 6 months and then yearly to five years) with yearly CT scanning and regular $\mathrm{X}$-ray mammography surveillance until 10 years post surgery. Information on date and cause of death was checked with that received by the Cancer Registry system and the Registrar General (Scotland).

Statistical analysis. The inter-observer interclass correlation coefficient was performed using Reliability analysis. Inter-relationships between variables were assessed using contingency table analysis with the chi-squared test for trend as appropriate. As there appears to be no consistent and well defined cutoffs for CD4+ T-lymphocytic infiltrate, CD8 + T-lymphocytic infiltrate, CD20 + B-lymphocytic infiltrate and CD138 + B-lymphocytic infiltrate, the data were grouped into tertiles $(0,1-34$ and $\geqslant 35)$ for $\mathrm{CD} 4+$ T-lymphocytic infiltrate, $(\leqslant 4,5-39$ and $\geqslant 40)$ for CD8 + T-lymphocytic infiltrate, $(0,1-12$ and $\geqslant 13)$ for CD20 + B-lymphocytic infiltrate and $(0,1-9$ and $\geqslant 10)$ for CD138+ B-lymphocytic infiltrate (Figure 1). On inspection of the survival curves for these tertiles (Figure 2A-D), there was no association between CD4 + T-lymphocytic infiltrate, CD20 + B-lymphocytic infiltrate and cancer-specific survival (Figure 2A and $\mathrm{C}$ ), there was a significant association between CD8 + T-lymphocytic infiltrate and improved cancer-specific survival (Figure $2 \mathrm{~B}$ ) and there was significant association between CD138 + B-lymphocytic infiltrate
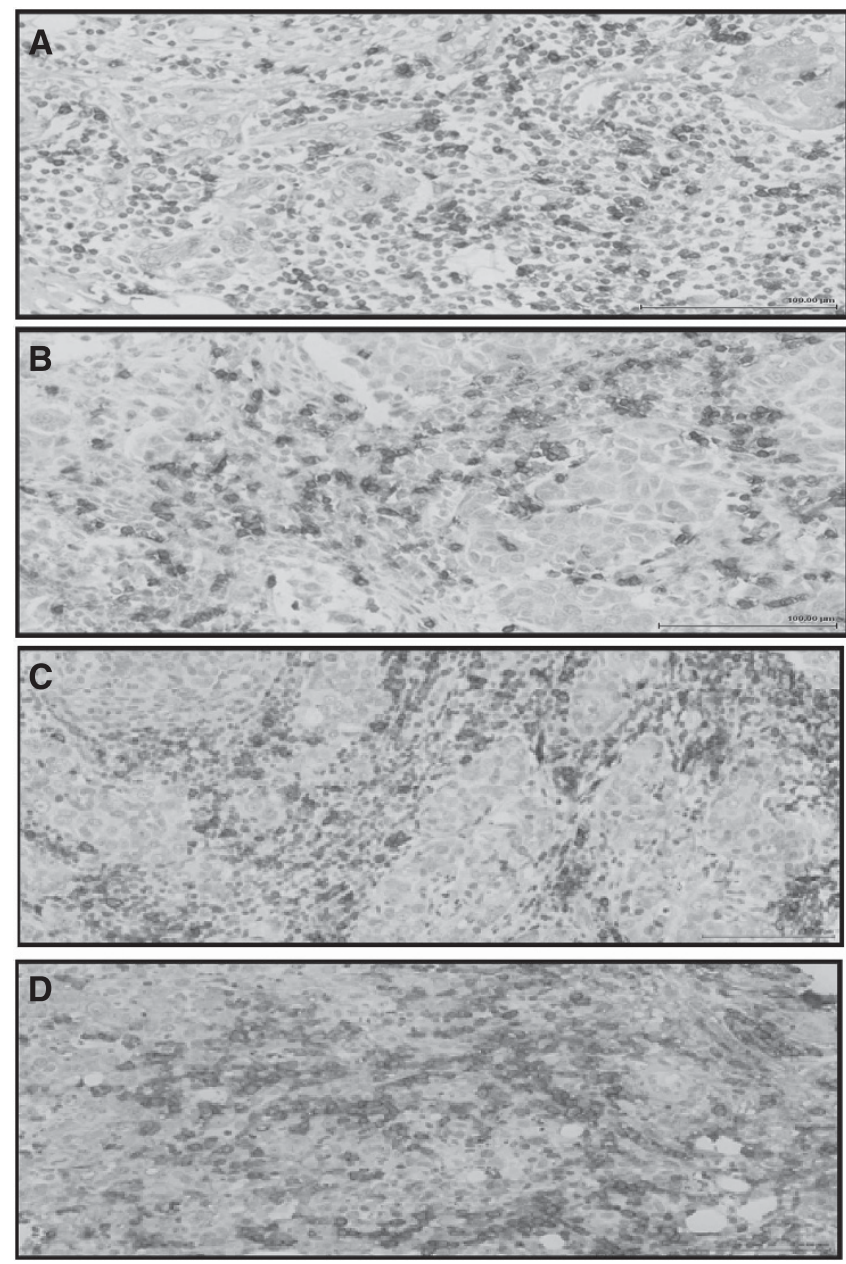

Figure 1. (A) CD4 + , (B) CD8 + , (C) CD20 + and (D) CD138+ immunohistochemical staining in invasive ductal breast cancer $(\times 20)$. 
and poorer cancer-specific survival (Figure 2D). Also, the defined and validated cutoffs for CD8 + (Baker et al, 2011; Mahmoud et al, 2011b; Liu et al, 2012) and CD20 + (Mahmoud et al, 2011a) alongside the tertiles were used in the current analysis.
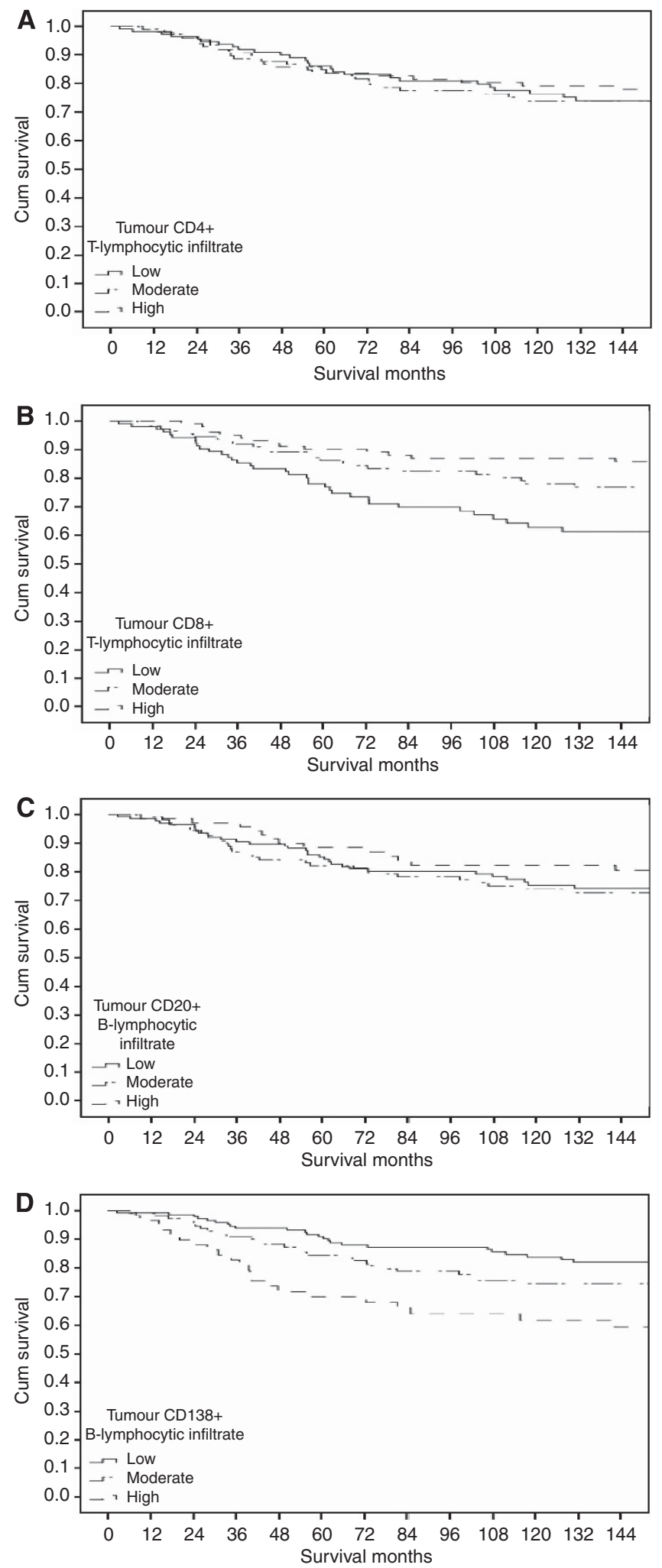

Figure 2. The relationship between CD4 + T-lymphocytic infiltrate (A), CD8 + T-lymphocytic infiltrate (B), CD20 + B-lymphocytic infiltrate (C) and CD138 + B-lymphocytic infiltrate (D) and cancer-specific survival in all patients with invasive ductal breast cancer.
Univariate and multivariate survival analysis with calculation of hazard ratios (HR) and 95\% confidence interval (95\% CI) were performed using a Cox proportional-hazards model. A stepwise backward procedure was used to derive a final model of the variables that had a significant independent relationship with survival. During this analysis, the proportionality assumptions in the Cox model were explored using a log minus log visual inspection and were found to be satisfactory. The relationship between the clinico-pathological characteristics and cancer-specific survival was also assessed using Kaplan-Meier plots. No variable examined in such analysis had more than $17 \%$ of total observations missing. Deaths up to March 2010 were included in the analysis. Because of the number of statistical comparisons, a $P$-value $<0.01$ was considered to be significant. All statistical analysis was performed using SPSS software version 19 (SPSS Inc., Chicago, IL, USA)

\section{RESULTS}

Baseline clinico-pathological characteristics of the patients with primary operable ductal invasive breast cancer $(n=338)$ are shown in Table 1.

Relationships between generalised inflammatory cells infiltrate and clinico-pathological characteristics are shown in Table 2. Patients with high-grade tumour inflammatory cell infiltrate (91\%) had higher tumour grade $(P<0.05)$, more ER-negative tumours $(P<0.01)$, more PR-negative tumours $(P<0.05)$, higher percentage

Table 1. Clinico-pathological characteristics of patients with primary operable invasive ductal breast $(n=338)$

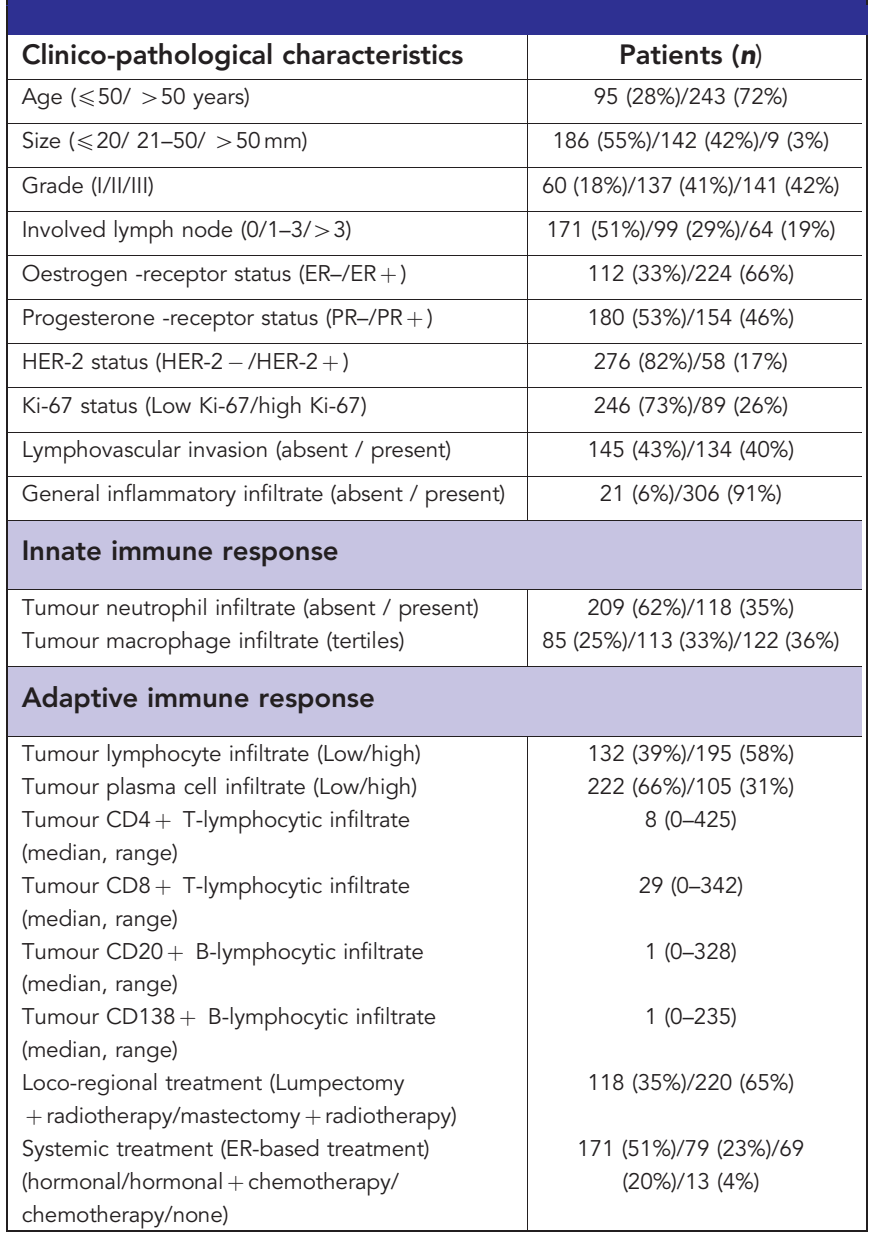


of neutrophil cell infiltrate at the invasive margin $(P<0.01)$, higher number of macrophage cell infiltrate at the invasive margin $(P<0.001)$, higher percentage of lymphocyte cell infiltrate at the invasive margin $(P<0.001)$, higher percentage of plasma cell infiltrate at the invasive margin $(P<0.01)$, higher number of CD8 + T-lymphocytic infiltrate $(P<0.01)$, higher number of $\mathrm{CD} 20+$ T-lymphocytic infiltrate $(P<0.01)$ and had a longer cancer-specific survival $(P<0.01)$.

In order to account for the potential confounding effect of the extent of inflammatory infiltrate on the relationship of lymphocyte subsets and survival, the subsequent analysis was performed in patients who had a significant inflammatory infiltrate.

Minimum follow-up was 26 months; the median follow-up of the survivors was 164 months. In the patients with inflammatory infiltrate 73 patients developed recurrence, 14 local, 44 distant and 4 both, 144 patients died, 65 from their disease.

The relationship between clinico-pathological characteristics of patients with primary operable invasive ductal breast cancer and had high-grade inflammatory infiltrate and recurrence-free survival was examined. On univariate survival analysis, tumour neutrophil infiltrate (HR: $1.89,95 \% \mathrm{CI}: 1.12-3.19, P=0.016$ ), tumour plasma cell infiltrate (HR: 2.96, 95\% CI: 0.17-0.50, $P<0.001)$ and tumour $\mathrm{CD} 138+$ B-lymphocytic infiltrate (HR: $1.92,95 \%$ CI: $1.37-2.68, P<0.001$ ) were significantly associated with poorer recurrence-free survival. Tumour lymphocytic infiltrate (HR: $0.30,95 \%$ CI: $0.17-0.51, \quad P<0.001$ ), lymphocyte infiltrate/ tumour plasma cell infiltrate ratio (HR: 0.51, 95\% CI: 0.35-0.74, $P<0.001)$, tumour CD8 + T-lymphocytic infiltrate (tertiles) (HR: $0.67,95 \% \mathrm{CI}: 0.48-0.93, P=0.018$ ) and tumour CD8 + T-lymphocyte infiltrate/ tumour CD138 + B-lymphocytic infiltrate ratio (HR: $0.39,95 \% \mathrm{CI}: 0.26-0.59, P<0.001$ ) were significantly associated with improved recurrence-free survival. On multivariate survival analysis, tumour CD8 + T-lymphocyte infiltrate/ tumour $\mathrm{CD} 138+$ B-lymphocytic infiltrate ratio (HR: $0.45,95 \%$ CI: $0.29-0.70, P<0.001$ ) was independently associated with improved recurrence-free survival and tumour plasma cell infiltrate (HR: 2.70, 95\% CI: $1.30-5.58, P=0.007$ ) was significantly associated with poorer recurrence-free survival. When the established pathological prognostic factors including age, tumour size, grade, lymph node status, PR status, HER-2 status, Ki-67 proliferative activities and lymphovascular invasion were included in the multivariate model, lymphovascular invasion (HR: 4.86, 95\% CI: 2.10-11.24, $P<0.001$ ) and plasma cell infiltrate (HR: $2.43,95 \%$ CI: $1.22-4.82, \quad P=0.011$ ) were significant independent predictors of poorer recurrence-free survival. Tumour CD8 + T-lymphocyte infiltrate/ tumour CD138 + B-lymphocytic infiltrate ratio (HR: $0.40,95 \% \mathrm{CI}: 0.26-0.62, P<0.001$ ) was significant independent predictor of improved recurrence-free survival for patients with high-grade inflammatory infiltrate primary operable invasive breast cancer.

The relationships between clinico-pathological characteristics of patients primary operable invasive breast cancer and had highgrade inflammatory infiltrate and cancer-specific survival are shown in Table 3. On univariate survival analysis, tumour macrophage infiltrate $(P<0.05)$, tumour lymphocytic infiltrate $(P<0.001)$, tumour lymphocyte infiltrate/ tumour plasma cell

Table 2. The relationship between general inflammatory infiltrate and clinico-pathological characteristics of patients with primary operable invasive ductal breast cancer

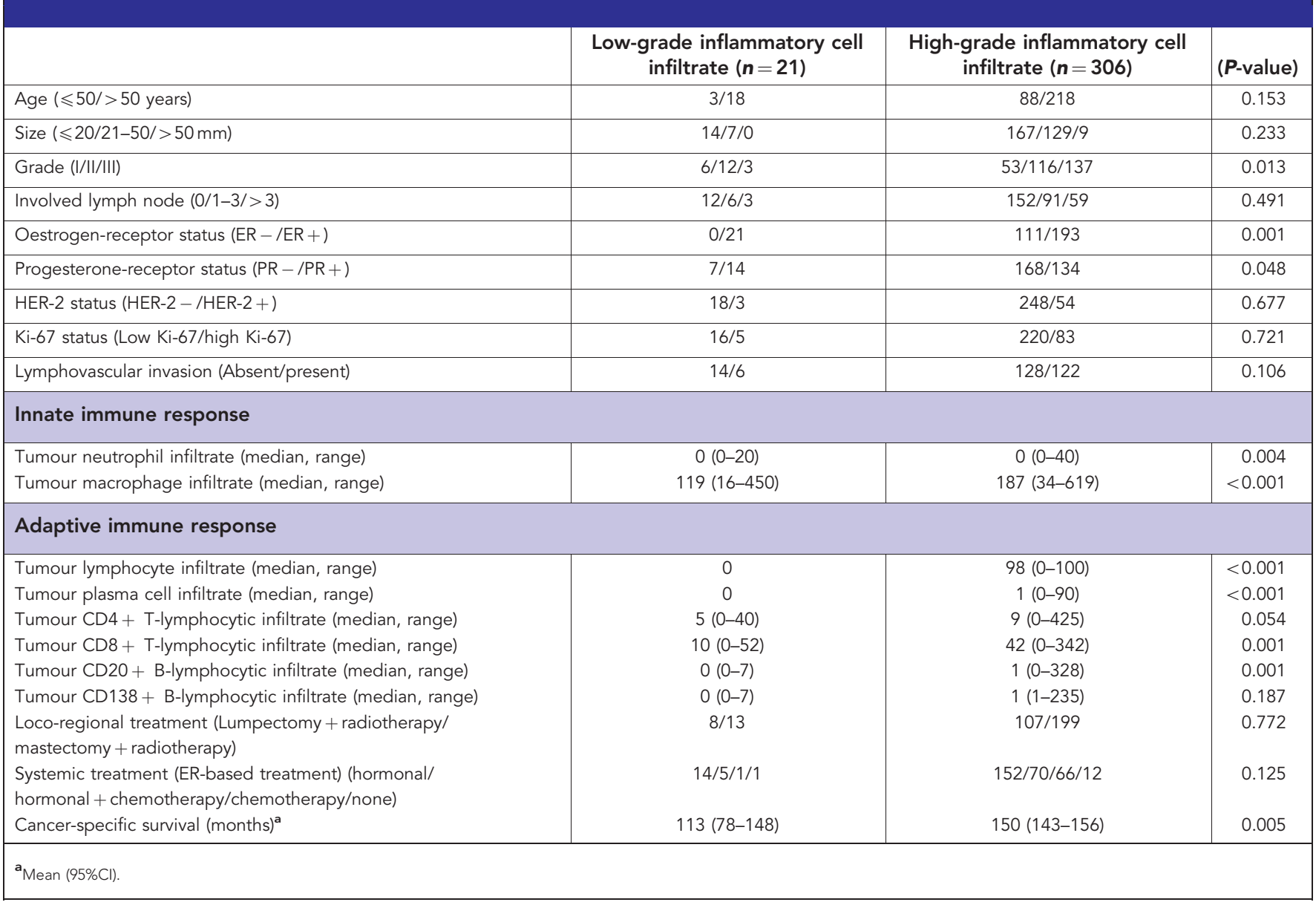


infiltrate ratio $(P<0.001)$, tumour CD8 + T-lymphocytic infiltrate (tertiles) and tumour CD8 + T-lymphocyte infiltrate/ tumour $\mathrm{CD} 138+\mathrm{B}$-lymphocytic infiltrate ratio $(P<0.001)$ were significantly associated with improved cancer-specific survival. Tumour neutrophil infiltrate $(P<0.05)$, tumour plasma cell infiltrate $(P<0.001)$ and tumour $\mathrm{CD} 138+$ B-lymphocytic infiltrate $(P<0.001)$ were significantly associated with poorer cancerspecific survival. On multivariate survival analysis, lymphocytic infiltrate $(P<0.001)$ and tumour CD8 + T-lymphocyte infiltrate/ tumour $\mathrm{CD} 138+\mathrm{B}$-lymphocytic infiltrate ratio $(P<0.001)$ were independently associated with improved cancer-specific survival. When the established pathological prognostic factors including age, tumour size, grade, lymph node status, PR status, HER-2 status, Ki-67 proliferative activities and lymphovascular invasion were included in the multivariate model (Table 4), lymphovascular invasion $(P<0.001)$ was significant independent predictors of poorer cancer-specific survival, and lymphocytic infiltrate $(P<0.001)$ and tumour CD8 + T-lymphocyte infiltrate/ tumour $\mathrm{CD} 138+\mathrm{B}$-lymphocytic infiltrate ratio $(P<0.001)$ were significant independent predictors of improved cancer-specific survival for patients with high-grade inflammatory infiltrate primary operable invasive breast cancer.

The inter-relationships between the different inflammatory cell types and clinico-pathological characteristics for patients with primary operable invasive ductal breast cancer are shown in Table 5. Tumour grade was directly associated with tumour macrophage infiltrate $(P<0.001)$ and $\mathrm{CD} 8+$ and $\mathrm{CD} 138+$ infiltrates (all $P<0.001$ ) and inversely associated with the percentage of tumour lymphocyte infiltrate $(P<0.001)$. The expression of ER was directly associated with the percentage of tumour lymphocyte infiltrate $(P<0.001)$ and inversely associated with $\mathrm{CD} 8+(P<0.01)$ and $\mathrm{CD} 138+$ infiltrates $(P<0.001)$. The expression of $\mathrm{PR}$ was directly associated with the percentage of tumour lymphocyte infiltrate $(P<0.001)$ and inversely associated with CD138 + infiltrates $(P<0.01)$. The expression of HER-2 was directly associated with $\mathrm{CD} 8+(P<0.001)$ and inversely associated with $\mathrm{CD} 138+$ infiltrates $(P<0.001)$. Tumour macrophage infiltrate was directly associated with tumour CD8 $+(P<0.001)$ and $\mathrm{CD} 138+(P<0.01)$ infiltrates. Tumour CD8 + T-lymphocyte infiltrate was directly associated with $\mathrm{CD} 138+(P<0.001)$ infiltrates.

\section{DISCUSSION}

The results of the present study show that the majority (91\%) of patients with primary operable invasive ductal breast cancer had high-grade inflammatory infiltrate and that most inflammatory cell types were greater in number compared with a low-grade inflammatory cell infiltrate. In particular, adaptive immune response components especially $\mathrm{CD} 8+\mathrm{T}$ lymphocytes were greater in number. For example, the majority of T-lymphocytes were $\mathrm{CD} 8+\mathrm{T}$ lymphocytes rather than $\mathrm{CD} 4+\mathrm{T}$ lymphocytes as might be expected from their circulating numbers (the CD $4+1$ CD8 + ratio was $1: 3$ in contrast to the expected ratio of $2: 1$ ). When the prognostic value of the lymphocyte subsets were examined in multivariate survival analysis, it was of interest that $\mathrm{CD} 8+$ and $\mathrm{CD} 138+$ infiltration displaced tumour-based factors including grade and hormone status as prognostic factors. Taken together, these results would indicate that the majority of patients with primary operable invasive ductal breast cancer have evidence of a high-grade inflammatory cell infiltrate and that components of this response, representing a coordinated immune response, have prognostic value superior to that of tumour-based factors.

With reference to the present observation that a high CD138+ B-lymphocytic infiltrate was associated with poorer cancer survival, it is well recognised that B lymphocytes contribute to cellular immunity by serving as antigen-presenting cells (APCs) and/or by providing co-stimulatory signals to T-lymphocytes (Crawford et al, 2006). Therefore, it may be that inappropriately large numbers of such cells promote tumour progression or alternatively that large highly proliferating tumours bring about downregulation and upregulation of $\mathrm{T}$ - and B-lymphocytic responses, respectively. However, the former explanation is a more likely as neither cell type was significantly associated with tumour size of the Ki-67-labelling index and that both cell types were associated with grade. Indeed, chronic activation of B-lymphocytes can be deleterious, as evidenced by their association with several pathologic disease states (rheumatoid arthritis and other autoimmune diseases) and some cancer types (DeNardo and Coussens, 2007; Youinou et al, 2007).

The basis of the relationship between tumour grade, tumour macrophage, $\mathrm{CD} 8+$ and $\mathrm{CD} 138+$ infiltrates and cancer outcome

Table 3. The relationship between components of inflammatory cell infiltrate of patients with high grade inflammatory cell infiltrate primary operable invasive ductal breast cancer and cancer-specific survival

Univariate survival analysis

Multivariate survival analysis

\begin{tabular}{|c|c|c|c|c|}
\hline & Hazard ratio $(95 \% \mathrm{Cl})$ & $\boldsymbol{P}$-value & $\begin{array}{c}\text { Hazard ratio } \\
(95 \% \mathrm{Cl})\end{array}$ & $\boldsymbol{P}$-value \\
\hline \multicolumn{5}{|l|}{ Innate immune response } \\
\hline Tumour neutrophil infiltrate (Low/high) & $1.78(1.09-2.90)$ & 0.020 & & 0.800 \\
\hline Tumour macrophage infiltrate (tertiles) & $0.71(0.51-0.98)$ & 0.035 & & 0.170 \\
\hline \multicolumn{5}{|l|}{ Adaptive immune response } \\
\hline Tumour lymphocyte infiltrate (Low/high) & $0.13(0.07-0.23)$ & $<0.001$ & $0.21(0.20-0.47)$ & $<0.001$ \\
\hline Tumour plasma cell infiltrate (Low/high) & $5.54(3.24-9.46)$ & $<0.001$ & & 0.956 \\
\hline Tumour lymphocyte infiltrate/Tumour plasma cell infiltrate ratio (tertiles) & $0.33(0.22-0.48)$ & $<0.001$ & & 0.330 \\
\hline Tumour CD4 + T-lymphocytic infiltrate (tertiles) & $0.96(0.71-1.29)$ & 0.780 & & \\
\hline Tumour CD8 + T-lymphocytic infiltrate $(<2 / \geqslant 2)$ & $0.89(0.32-2.43)$ & 0.813 & & \\
\hline Tumour CD8 + T-lymphocytic infiltrate (tertiles) & $0.55(0.40-0.76)$ & $<0.001$ & & 0.597 \\
\hline Tumour CD20 + B-lymphocytic infiltrate (>5) & $0.85(0.50-1.45)$ & 0.555 & & \\
\hline Tumour CD20 + B-lymphocytic infiltrate (tertiles) & $0.91(0.66-1.24)$ & 0.534 & & \\
\hline Tumour CD138 + B-lymphocytic infiltrate (tertiles) & $1.92(1.41-2.62)$ & $<0.001$ & & 0.422 \\
\hline Tumour CD8 + infiltrate/Tumour CD138 + infiltrate ratio (tertiles) & $0.40(0.27-0.59)$ & $<0.001$ & $0.46(0.30-0.70)$ & $<0.001$ \\
\hline
\end{tabular}


Table 4. The relationship between clinic-pathological characteristics of patients with high-grade inflammatory cell infiltrate primary operable invasive ductal breast cancer and cancer-specific survival

\begin{tabular}{|c|c|c|c|c|}
\hline & \multicolumn{2}{|c|}{ Univariate survival analysis } & \multicolumn{2}{|c|}{ Multivariate survival analysis } \\
\hline & Hazard ratio $(95 \% \mathrm{Cl})$ & $P$-value & Hazard ratio $(95 \% \mathrm{Cl})$ & $\boldsymbol{P}$-value \\
\hline Age $(<50 />50$ years $)$ & $1.43(0.82-2.52)$ & 0.211 & & \\
\hline Size $(\leqslant 20 / 21-50 />50 \mathrm{~mm})$ & $2.57(1.67-3.95)$ & $<0.001$ & & 0.064 \\
\hline Grade (I/II/IIII) & $1.84(1.25-2.68)$ & 0.002 & & 0.299 \\
\hline Involved lymph node $(0 / 1-3 />3)$ & $2.45(1.80-3.33)$ & $<0.001$ & & 0.255 \\
\hline Oestrogen-receptor status status (ER - /ER +) & $0.41(0.25-0.66)$ & $<0.001$ & & 0.956 \\
\hline Progesterone-receptor status (PR - /PR +) & $0.35(0.20-0.61)$ & $<0.001$ & & 0.713 \\
\hline HER-2 status (HER-2 - /HER-2 +) & $1.66(0.93-2.95)$ & 0.086 & & 0.678 \\
\hline Ki-67/ MIB1 proliferation index (Low Ki-67/high Ki-67) & $1.76(1.06-2.91)$ & 0.028 & & 0.371 \\
\hline Lymphovascular invasion (Absent/present) & $4.82(2.48-9.34)$ & $<0.001$ & $5.66(1.92-16.65)$ & 0.002 \\
\hline \multicolumn{5}{|l|}{ Innate immune response } \\
\hline Tumour macrophage infiltrate (tertiles) & $0.71(0.51-0.98)$ & 0.035 & & 0.056 \\
\hline \multicolumn{5}{|l|}{ Adaptive immune response } \\
\hline $\begin{array}{l}\text { Tumour lymphocyte infiltrate (Low/high) } \\
\text { Tumour lymphocyte infiltrate/tumour plasma cell infiltrate ratio (tertiles) } \\
\text { Tumour CD8 + T-lymphocytic infiltrate (tertiles) } \\
\text { Tumour CD138 + B-lymphocytic infiltrate (tertiles) } \\
\text { Tumour CD8 + infiltrate/tumour CD138 + infiltrate ratio (tertiles) }\end{array}$ & $\begin{array}{l}0.13(0.07-0.23) \\
0.33(0.22-0.48) \\
0.55(0.40-0.76) \\
1.92(1.41-2.62) \\
0.40(0.27-0.59)\end{array}$ & $\begin{array}{l}<0.001 \\
<0.001 \\
<0.001 \\
<0.001 \\
<0.001\end{array}$ & $0.21(0.09-0.50)$ & $\begin{array}{r}<0.001 \\
0.245 \\
0.851 \\
0.194 \\
0.002\end{array}$ \\
\hline
\end{tabular}

is not clear. One hypothesis would be that the tumour necrosis may have part in these relationships as rapidly growing tumours quickly outgrow their own blood supply, becoming necrotic, and this results in the production of stress protein including chemokines and cytokines that attract specific inflammatory cells. Indeed, Carter et al (1978) and Carlomagno et al (1995) reported that tumours with extensive necrosis contained more inflammatory cells . Moreover, Richards et al (2012) reported that the impact of tumour necrosis on colorectal cancer survival may be explained by close relationships with host inflammatory responses. Therefore, further work is required to examine the relationship between tumour necrosis, the nature of the inflammatory cell infiltrate and survival in patients with primary operable invasive breast cancer.

The results of the present study build on our previous work using H\&E (Mohammed et al, 2012b) and indicate that the ratio between the lymphocytic/plasma cell infiltrate using more sophisticated immunohistochemical techniques has an independent prognostic role, superior to individual inflammatory cell types. If this work was to be confirmed in other cohorts it would enable the definition of a reliable scoring system to help rationalise the enormous amount of work that has and is continuing to be carried out into the nature of the inflammatory cell infiltrate in patients with breast cancer. This ratio can be readily standardised, using automated counting systems, and can be carried out in both H\&E slides and also IHC slides. Finally it may provide a useful therapeutic target.

The results of the present study may also shed some light on the nature of the local inflammatory response, as they would suggest that a coordinated response between lymphocytes and plasma cells might have a profound effect on outcome. Indeed, it is well recognised that $\mathrm{B}$ lymphocytes contribute to cellular immunity by serving as antigen-presenting cells (APCs) and/or by providing costimulatory signals to T lymphocytes (Crawford et al, 2006). For example, as the ratio of CD8 T-lymphocytes to CD138 B-lymphocytes increased (from $\leqslant 3$ to $4-9$ to $\geqslant 10$ ), there was an approximately five-fold improvement in recurrence-free and cancer-specific survival. These results may suggest that a dysregulated immune response with downregulation and upregulation of T- and B-lymphocytic responses, respectively (DeNardo and Coussens, 2007; Youinou et al, 2007).

There are striking similarities to recent work in colorectal cancer that has resulted in a profusion of immune-based prognostic scores (Galon et al, 2006; Bindea et al, 2011). With reference to breast cancer, DeNardo et al (2011) have proposed the combination of a low CD68 + and low CD4 + and high CD8 + for predicting improved survival in patients with operable breast cancer. The results of the present study, in patients with a high grade inflammatory cell infiltrate, confirm the utility of high CD8+ T-lymphocytic infiltrate but not a low CD68 + macrophage infiltrate or a low $\mathrm{CD} 4+$ T-lymphocytic infiltrate having independent associations with improved survival in patients with operable ductal breast cancer. Interestingly, a high CD138+ B-lymphocytic infiltrate was also shown to be associated with poorer outcome and had independent prognostic value. The significance of the present discrepant findings are as yet unclear. However, they do point clearly to the importance of immune context and the need to assess the extent of the inflammatory cell infiltrate before carrying out more sophisticated analysis on particular inflammatory cell types.

The present study has a number of limitations. In particular, the analysis of the inflammatory cell infiltrate was undertaken using visual quantitative and semi-quantitative techniques. To minimise the risk of measurement bias, two independent observers examined the same cores or sections before assigning an overall score. High levels of independent inter-observer agreement in the assessment of all examined factors suggest that these techniques are reliable. Also, the limitation of whether a small core sample used in the present TMA was representative of the entire tumour was examined, using a pilot comparison study of at least 30 full-faced breast cancer tissue sections. Also, in the present study, defined and validated thresholds for CD8 + and CD20 + were included to thoroughly examine their relationship with the survival. Clearly, 


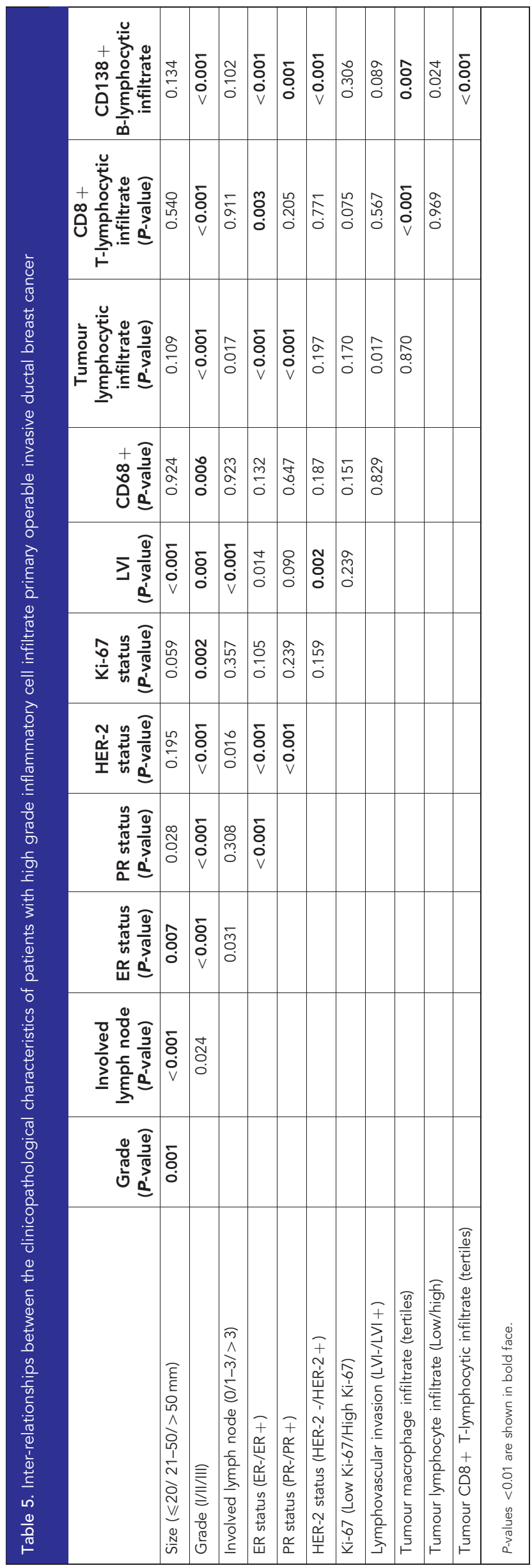

the novel findings of the present study require independent validation in other centres and in contemporary cohorts of patients.

In summary, the results of the present study indicate that, in ductal breast cancer, the majority of patients have a high-grade inflammatory cell infiltrate, there are relationships between tumour grade, tumour macrophage, CD8 + and CD138 + infiltrates and these inflammatory cell types are independent predictors of cancer survival in patients primary operable invasive ductal breast cancer. Further, the ratio between lymphocyte and plasma cell infiltrate ( $\mathrm{H} \& \mathrm{E}$ and $\mathrm{IHC}$ ) had independent prognostic value.

\section{ACKNOWLEDGEMENTS}

The authors gratefully acknowledged the funding from Libyan government. We also thank Clare Orange, who made and cut tissue microarray, Dr Elizabeth Mallon for marking the slides for tissue microarray and Dr Julie C Doughty for providing us the database.

\section{REFERENCES}

Baker K, Lachapelle J, Zlobec I, Bismar TA, Terracciano L, Foulkes WD (2011) Prognostic significance of CD8 $+\mathrm{T}$ lymphocytes in breast cancer depends upon both oestrogen receptor status and histological grade. Histopathology 58(7): 1107-1116.

Bindea G, Mlecnik B, Fridman WH, Galon J (2011) The prognostic impact of anti-cancer immune response: a novel classification of cancer patients. Semin Immunopathol 33(4): 335-340.

Cancerstats (2011) 25-6-2012. Ref type: Online Source.

Carlomagno C, Perrone F, Lauria R, de Laurentiis M, Gallo C, Morabito A, Pettinato G, Panico L, Bellelli T, Apicella A (1995) Prognostic significance of necrosis, elastosis, fibrosis and inflammatory cell reaction in operable breast cancer. Oncology 52(4): 272-277.

Carter D, Pipkin RD, Shepard RH, Elkins RC, Abbey H (1978) Relationship of necrosis and tumor border to lymph node metastases and 10-year survival in carcinoma of the breast. Am J Surg Pathol 2(1): 39-46.

Colotta F, Allavena P, Sica A, Garlanda C, Mantovani A (2009) Cancer-related inflammation, the seventh hallmark of cancer: links to genetic instability. Carcinogenesis 30(7): 1073-1081.

Costes V, Magen V, Legouffe E, Durand L, Baldet P, Rossi JF, Klein B, Brochier J (1999) The Mi15 monoclonal antibody (anti-syndecan-1) is a reliable marker for quantifying plasma cells in paraffin-embedded bone marrow biopsy specimens. Hum Pathol 30(12): 1405-1411[pii].

Crawford A, Macleod M, Schumacher T, Corlett L, Gray D (2006) Primary $\mathrm{T}$ cell expansion and differentiation in vivo requires antigen presentation by B cells. J Immunol 176(6): 3498-3506[pii].

DeNardo DG, Brennan DJ, Rexhepaj E, Ruffell B, Shiao SL, Madden SF, Gallagher WM, Wadhwani N, Keil SD, Junaid SA, Rugo HS, Hwang ES, Jirstroem K, West BL, Coussens LM (2011) Leukocyte complexity predicts breast cancer survival and functionally regulates response to chemotherapy. Cancer Discovery 1(1): 54-67.

DeNardo DG, Coussens LM (2007) Inflammation and breast cancer. Balancing immune response: crosstalk between adaptive and innate immune cells during breast cancer progression. Breast Cancer Res 9(4): 212.

Galon J, Costes A, Sanchez-Cabo F, Kirilovsky A, Mlecnik B, Lagorce-Pages C, Tosolini M, Camus M, Berger A, Wind P, Zinzindohoue F, Bruneval P, Cugnenc PH, Trajanoski Z, Fridman WH, Pages F (2006) Type, density, and location of immune cells within human colorectal tumors predict clinical outcome. Science 313(5795): 1960-1964[pii].

Hanahan D, Weinberg RA (2011) Hallmarks of cancer: the next generation. Cell 144(5): 646-674.

Klintrup K, Makinen JM, Kauppila S, Vare PO, Melkko J, Tuominen H, Tuppurainen K, Makela J, Karttunen TJ, Makinen MJ (2005) Inflammation and prognosis in colorectal cancer. Eur J Cancer 41(17): 2645-2654.

Lal P, Tan LK, Chen B (2005) Correlation of HER-2 status with estrogen and progesterone receptors and histologic features in 3,655 invasive breast carcinomas. Am J Clin Pathol 123(4): 541-546. 
Liu S, Lachapelle J, Leung S, Gao D, Foulkes WD, Nielsen TO (2012) CD8 + lymphocyte infiltration is an independent favorable prognostic indicator in basal-like breast cancer. Breast Cancer Res 14(2): R48.

Mahmoud SM, Lee AHS, Paish EC, Macmillan RD, Ellis IO, Green AR (2011a) The prognostic significance of B lymphocytes in invasive carcinoma of the breast. Breast Cancer Res Treat 132(2): 545-553.

Mahmoud SM, Paish EC, Powe DG, Macmillan RD, Grainge MJ, Lee AHS, Ellis IO, Green AR (2011b) Tumor-infiltrating CD8 + lymphocytes predict clinical outcome in breast cancer. J Clin Oncol 29(15): 1949-1955.

Mohammed ZM, Edwards J, Orange C, Mallon E, Doughty JC, McMillan DC, Going JJ (2012a) Breast cancer outcomes by steroid hormone receptor status assessed visually and by computer image analysis. Histopathology 61(2): 283-292.

Mohammed ZM, Going JJ, Edwards J, Elsberger B, Doughty JC, McMillan DC (2012b) The relationship between components of tumour inflammatory cell infiltrate and clinicopathological factors and survival in patients with primary operable invasive ductal breast cancer. Br J Cancer 107(5): 864-873.

Mohammed ZM, Going JJ, Edwards J, McMillan DC (2012c) The role of the tumour inflammatory cell infiltrate in predicting recurrence and survival in patients with primary operable breast cancer. Cancer Treat Rev 38(8): 943-955.

Mohammed ZM, Going JJ, McMillan DC, Orange C, Mallon E, Doughty JC, Edwards J (2012d) Comparison of visual and automated assessment of
HER2 status and their impact on outcome in primary operable invasive ductal breast cancer. Histopathology 61(4): 675-684.

Mohammed ZM, McMillan DC, Elsberger B, Going JJ, Orange C, Mallon E, Doughty JC, Edwards J (2012e) Comparison of Visual and automated assessment of Ki-67 proliferative activity and their impact on outcome in primary operable invasive ductal breast cancer. $B r J$ Cancer 106(2): 383-388.

Parkin DM, Bray F, Ferlay J, Pisani P (2001) Estimating the world cancer burden: Globocan 2000. Int J Cancer 94(2): 153-156.

Richards CH, Roxburgh CS, Anderson JH, McKee RF, Foulis AK, Horgan PG, McMillan DC (2012) Prognostic value of tumour necrosis and host inflammatory responses in colorectal cancer. Br J Surg 99(2): 287-294.

Tedder TF, Engel P (1994) CD20: a regulator of cell-cycle progression of B lymphocytes. Immunol Today 15(9): 450-454.

Vakkila J, Lotze MT (2004) Inflammation and necrosis promote tumour growth. Nat Rev Immunol 4(8): 641-648.

Youinou P, Jamin C, Saraux A (2007) B-cell: a logical target for treatment of rheumatoid arthritis. Clin Exp Rheumatol 25(2): 318-328.

This work is published under the standard license to publish agreement. After 12 months the work will become freely available and the license terms will switch to a Creative Commons AttributionNonCommercial-Share Alike 3.0 Unported License. 